\title{
Role of Contrast Enhanced Ultrasound (CEUS) in the Paediatric Population With Blunt Abdominal Trauma: a Prospective Single Center Case Series
}

\section{Savvas Deftereos}

Democritus University of Thrace

Konstantinos Skarentzos ( $\square$ k.skarentzos@gmail.com )

Democritus University of Thrace

Soultana Foutzitzi

University Hospital of Alexandroupolis

Maria Aggelidou

University Hospital of Alexandroupolis

Panagoula Oikonomou

Democritus University of Thrace

Katerina Kambouri

Democritus University of Thrace

\section{Research Article}

Keywords: abdominal injury, BAT, CECT, CEUS, child

Posted Date: June 1st, 2021

DOI: https://doi.org/10.21203/rs.3.rs-558555/v1

License: (1) This work is licensed under a Creative Commons Attribution 4.0 International License.

Read Full License 


\section{Abstract}

Aim: The aim of our study is to evaluate the diagnostic ability of contrast-enhanced ultrasonography (CEUS) in pediatric population with history of blunt abdominal trauma (BAT).

Materials and Methods: In an eight-year period (1/2012-1/2020) fifty-nine children (4-14 years old) were transferred to Emergency Department with referred BAT. The initial imaging method was ultrasound scan (US). Thirty-two children were discharged in good condition 24/hours after their admission. The rest 27 with moderate to severe injuries and according to their laboratory tests and US results, were evaluated with CEUS and contrast-enhanced computed tomography (CECT).

Results: Five children were confirmed with splenic injury, two with liver lacerations, one with liver lacerations and right kidney contusion, while in nineteen, no visceral pathology was found by US, CEUS and CECT. The CEUS and CECT were in complete agreement. In contrary, unenhanced-US showed in eleven children free peritoneal fluid and in two possible parenchymal lesions. In two of the patients with negative US-study splenic contusions in CECT and CEUS were revealed. The CEUS study was also used as follow-up method. No adverse reactions were observed from CEUS contrast agent in all patients (27) follow-up in 1-week and in 6-months period. Furthermore, in 27 patients no adverse reactions were observed from CEUS contrast agent in a 1-year period.

Conclusion: CEUS is effective, easily performed, low cost and radiation free, imaging method. It is ideal both for initial and follow up evaluation of trauma and thus we encourage the usage of the method in paediatric BAT cases.

\section{Introduction}

Trauma is the most frequent cause of death in the population younger than 45 years. The abdomen is the second most common site of injury and it is well known that a prompt diagnosis can prevent patients from severe consequences [1, 2]. Focused Assessment with Sonography for Trauma (FAST) or e-FAST (extended-FAST), is usually the initial imaging method for evaluation especially for haemodynamically unstable patients who sustained blunt abdominal trauma. e-FAST is performed to detect haemothorax, pneumothorax, and haemoperitoneum but has major limitations and poor sensitivity $(41 \%-44 \%)$ in the direct depiction of solid abdominal organ lesions [3].

In haemodynamically stable patients with high-energy trauma, or when the haemodynamic stabilization occurs, contrast-enhanced computed tomography (CECT) is performed. CECT is the gold standard in the evaluation of the injured patient. Unfortunately, CECT has the disadvantage of radiation exposure [4].

CEUS is a relatively novel, radiation-free method alternative to CECT with the potential to identify abdominal solid organs lacerations but its use is off-label in children (The contrast agent SonoVueß has recently been approved by the FDA under the name of Lumason ${ }^{\circledR}$ to be used in hepatic investigations in adults and children). The contrast agents are neither nephro-, hepato- or cardio-toxic and do not require 
testing of renal function prior to examination as is necessary with other contrast media (for computed tomography). Furthermore, the use of CEUS in children is supported by clinical experience $[5,6]$.

For children with blunt abdominal trauma, physical examination plus FAST and CEUS as needed, seems to have reasonable sensitivity, specificity, and accuracy in detecting intra-abdominal injuries and may reduce the need for CT scans [3]. This present study aims to estimate and underline the usefulness of CEUS in the evaluation of paediatric blunt abdominal trauma

\section{Materials And Methods}

The study was conducted during an eight-year period, from January 2012 to January 2020, at Emergency Department of our University Hospital. Patients enrolled in the study included all children up to 14 years old who were admitted for referred BAT and had positive clinical, laboratory and/or US findings. Research was performed in accordance with the Declaration of Helsinki. The research was approved by ethical and deontology committee (Protocol number: 21234) of General University Hospital of Alexandroupolis and science committee of our hospital. Informed consent was obtained from all participants and/or their legal guardians.

All the imaging methods were performed by two radiologists (one pediatric radiologist and one general) and the CEUS scans were followed by CECT within 30 minutes. All parents were informed about the imaging method (CEUS) and signed a consent form (deemed "off-label" method). Moreover, parents/guardians were informed that their children will be monitored for possible side effects after CEUS for about a year period. All CEUS scans were compared with CECT results as CECT is the gold standard in the evaluation of the injured patient [4].

During the eight-year study period, 59 children with age ranged from 4 to 14 years old (38 boys and 21 girls) were referred to the Emergency Department of our University Hospital with reported abdominal injury. These patients initially underwent conventional US that followed FAST. Thirty-two of them (21 boys and 11 girls) with minor injuries, normal physical examination, negative US and laboratory results within normal limits were discharged after 24 hours observation without undergoing further investigation. The rest twenty-seven patients, which form our study population, suffered from more severe injuries with positive clinical, laboratory and/or US findings (Table 1). 
Table 1

demographics and outcomes

\begin{tabular}{|c|c|c|c|c|c|}
\hline & age & sex & US/FAST & CEUS & CECT \\
\hline 1 & 14 & male & + (fluid) & Liver laceration & Liver laceration \\
\hline 2 & 9 & female & + (fluid) & L lacer + R Kidney & L lacer + R Kidney \\
\hline 3 & 13 & male & + (fluid) & spleen contusion & spleen contusion \\
\hline 4 & 11 & female & - & spleen rapture & spleen rapture \\
\hline 5 & 8 & female & + (fluid) / spleen suspicion & - & - \\
\hline 6 & 11 & male & + (fluid) & - & - \\
\hline 7 & 14 & female & + (focal liver hypoattenuation) & spleen rapture & spleen rapture \\
\hline 8 & 14 & male & - & spleen contusion & spleen contusion \\
\hline 9 & 14 & male & + (fluid) & spleen contusion & spleen contusion \\
\hline 10 & 14 & female & + (fluid) / liver suspicion & - & - \\
\hline 11 & 9 & male & + (focal liver hypoattenuation) & Liver laceration & Liver laceration \\
\hline 12 & 8 & female & - & - & - \\
\hline 13 & 4 & male & - & - & - \\
\hline 14 & 12 & male & - & - & - \\
\hline 15 & 11 & male & - & - & - \\
\hline 16 & 13 & male & - & - & - \\
\hline 17 & 8 & female & - & - & - \\
\hline 18 & 13 & female & - & - & - \\
\hline 19 & 12 & male & - & - & - \\
\hline 20 & 14 & male & - & - & - \\
\hline 21 & 13 & female & - & - & - \\
\hline 22 & 8 & male & - & - & - \\
\hline 23 & 4 & female & - & - & - \\
\hline 24 & 12 & male & - & - & - \\
\hline 25 & 11 & female & - & - & - \\
\hline 26 & 13 & male & - & - & - \\
\hline
\end{tabular}




\begin{tabular}{|llllll|}
\hline & age & sex & US/FAST & CEUS & CECT \\
\hline 27 & 8 & male & - & - \\
\hline FAST = Focused Assessment with Sonography for Trauma, CEUS = Contrast Enhanced Ultrasound, \\
CECT = contrast-enhanced CT, $-=$ negative results
\end{tabular}

\section{Ultrasonography and Contrast-Enhanced Ultrasonography}

Patients initially underwent FAST examination, and in suspicious and stable patients CEUS and CECT was performed. The CEUS-exam started typically from the kidneys, continued with the liver and ended in the spleen (Fig. 1). In cases, with not an appropriate imaging effect a resumption of the method with second intravenous injection was followed. The overall time needed was up to $5 \mathrm{~min}$ for each contrast agent injection.

The CEUS procedure requires contrast medium injection and US machine suitable to CEUS software. All CEUS studies were performed with a 5-1 MHz convex array transducer in a Philips iU22 ultrasound machine. The contrast media which was used in our study was SonoVueTM (Bracco, Milano, Italy). SonoVueTM is an agent made of microbubbles stabilized by phospholipids and containing Sulphur hexafluoride $\left(\mathrm{SF}_{6}\right)$, an innocuous gas. The microbubbles have a diameter ranged from 1 micron to 7 microns ( 1 micron is equal to $0,0001 \mathrm{~cm}$ ). This contrast medium is blood-pool agent with a non-linear reverberation on US. The microbubbles remain intravascular and produce a non-linear harmonic response that can be separated from the tissue signal using contrast harmonic US (equipment's software). Our dosage schemes for SonoVueTM were adjusted to $0.03 \mathrm{~mL}$ per $\mathrm{kg}(0.03 \mathrm{~mL} / \mathrm{kg})$ per every intravenous injection. In generally SonoVueTM suspension was administered by syringe bolus using an existing peripheral vein in a maximum amount of $0.3 \mathrm{ml}$ for children weighing $<20 \mathrm{~kg}$ and $0.5 \mathrm{ml}$ for children weighing $\geq 20 \mathrm{~kg}$. This was followed by a 5 or $10 \mathrm{ml}$ normal saline flush respectively. Vital signs were monitored at 2 min intervals during the CEUS-study and also 30 mins and 1 hour after completion of contrast injection for possible adverse reaction symptoms. This monitoring was completed with the recording of other side effects such as nausea, vomiting, dizziness etc.

On baseline US if lesions were visible, they were recognized as a hypoechoic or hyperechoic alteration(s) within the organ. On CEUS, when lesions were present in solid organ were clearly visible in all patients. The lesions were depicted as a perfusion defect (demarcated hypoechoic area compared to the adjacent parenchyma) with ill-defined or well-defined margins with or without interruption of the organ profile and margins. If lack of perfusion of a part(s) of the organ was present an arterial lesion was suspected while the presence of microbubbles (reflections) outside of the lacerated organ was defined as an active bleeding.

The arterial phase (first $30 \mathrm{sec}$ ) of CEUS were captured as video movie while selective spots from other phases also were recorded. CECT was performed within 30 minutes with intravenous injection of a 
nonionic contrast agent $(1,5 \mathrm{ml} / \mathrm{kg})$ and during the arterial phase, the venous phase and a late-phase study (5-15 $\mathrm{min}$ ), with the latter performed if fluid collections were revealed to identify more accurately any active bleeding and/or urinoma.

Data were analyzed using Microsoft Excel. The sensitivity, specificity, positive and negative predictive value of conventional US and CEUS were determined compared to CECT.

\section{Results}

The mean age of our study population was $10.93 \pm 2.9$ (mean \pm SD) years. Seventeen were boys $(63 \%)$ and ten were girls (37\%) (Table 1). The mechanisms of trauma in these children were 19 bicycle crashes (one bicycle vs car), 4 car accidents (one car vs pedestrian), 3 jumps from a high wall and one (1) slip in the bathtub. With the suspicion of visceral organ injury all these patients underwent CEUS-scan followed by CECT before their hospitalization (Table 1 ).

All children were in stable haemodynamic condition. No visceral pathology was found in 19 out of 27 children that were included in this study. The presence of fluid in the baseline US was accepted as an indirect evidence of visceral injury. According to that, US has high sensitivity and specificity $(80 \%$ and $84,21 \%$ respectively). On the other hand, the presence on US of hyperechoic lesion in visceral parenchyma has low sensitivity but high specificity for visceral injury ( $25 \%$ and $94,12 \%$ respectively).

In five children ( 3 boys and 2 girls average age 13.2 years) splenic injury (three contusions and two ruptures) was found. The mechanisms of accident were bicycle crash for two boys, jump of a high wall for one boy and one girl, and slip in the bathtub for one girl. Liver laceration was revealed in two boys and in one girl both liver laceration and right kidney contusion were depicted. The two boys ( 14 and 9 years old) with liver lacerations were involved in car accident and finally the 9-year-old girl was involved in a bicycle collision with car. CECT depicted the same lesions with CEUS. Contrarily, conventional US showed free peritoneal fluid in eleven patients and possible solid organ rupture (spleen and liver) in two (patient 5 and 10). In those two patients (patient 5 and 10) CEUS and CECT was administrated but no evidence of pathology was found. Even though, 18 children (patient 4, 8 and 12-27) had negative US, CECT and CEUS was conducted due to the severity of the mechanism of the injury and positive clinical and/or laboratory results (Fig. 2). In two children (patient 4 and 8), there were spleen contusions in CECT and CEUS. CECT and CEUS applied to eight children (patient 1-4, 7-9 and 11) for the follow up period and the results didn't differ from each other (Fig. 3). The examination with CEUS was held in less than ten minutes period without sedation and with lower cost than CECT scan. The patients were monitored for a year, the agent was well tolerated, and no adverse reactions were referred both at the early and late period.

\section{Discussion}


Trauma is the major cause of morbidity and mortality in pediatric population and the abdomen is the second most common site of injury [1]. The main focus of this study was the validation of CEUS in comparison with CT in identification possible visceral injuries in paediatric cases of Blunt Abdominal Trauma (BAT). According to our results, CEUS revealed the same outcomes with CECT. Regarding negatives and positives findings CEUS and CECT demonstrated similar results. On the contrary, US had 2 false negatives (7.41\%) (patient 4 and 8 ) and 3 false positives (11.11\%) (patient 5, 6 and 10). Even though, patients 4, 8 and 12-27 had negative US, we administrated CEUS and CECT because they had positive laboratory (first discovered-low hematocrit), or clinical results (e.g. abdominal pain). Although it seems like we overreacted, we were able to find two false negative US results (patient 4 and 8 ). Both pathologies (spleen rapture and spleen contusion) are medical emergencies. A misdiagnose in those patients might have proven fatal.

Diagnostic imaging plays an important role in the evaluation of trauma in children. The small body and the low amount of fat in children make them excellent candidates for conventional US which is widely used both for diagnostic and monitoring for many pediatric diseases. US use in paediatric trauma however is doubted. There are many reports that mention the conventional US's low sensitivity and unreliability as a diagnostic tool [7-10].

MRI in children despite its many advantages, it also has some disadvantages, such as the need for sedation or general anesthesia in younger children, the long exam duration and the use of gadoliniumbased contrast agents. Furthermore, in pediatric population, visceral organ bleeding tends to be selflimited despite the severity of trauma because there is a major vasoconstrictive response due to smaller blood vessels so in the management of the traumatized pediatric patient, a non-operative strategy is highly followed. Hence, after the initial evaluation (CECT and CEUS) and during follow-up, the injured child must be monitored according to radioprotection criteria (ALARA principles). Under these circumstances, CEUS can play a major role as a follow-up tool [11-13].

An important restriction of our study is the small number of children, and thus its limited ability to determine diagnostic accuracy in regard to specific organ injuries e.g. kidneys, liver. On other hand the sample size of splenic injuries was more frequent giving the possibility of safer conclusions. As it is well known, spleen is a well vascularized organ and in children is not protected from the rib cage like in adults because it is bigger. This is the reason why it is often involved in blunt abdominal trauma, with a frequency of $25 \%$ of the cases both as isolated or multiorgan lesions.

In hemodynamically stable trauma patients, paediatric patients included, CT is the imaging technique of choice to evaluate abdominal trauma. However, the benefit of avoiding ionizing radiation is far more important in children. Also, someone must keep in mind that the intravenous contrast used for CT is associated with up to a $4 \%$ incidence of adverse reactions [14].

\section{Conclusion}


Contrast-enhanced ultrasound is an imaging modality that can be used to diagnose the possibility of abdominal solid organ injury in paediatric blunt abdominal trauma without subjecting children to ionizing radiation. Of course, further clinical trials may aid in defining the ultimate utility of CEUS in paediatric BAT patients.

\section{Abbreviations}

BAT = blunt abdominal trauma

CECT $=$ contrast-enhanced ultrasonography

CEUS $=$ contrast-enhanced ultrasonography

$\mathrm{ED}=$ Emergency department

e-FAST $=$ extended-FAST

FAST $=$ Focused Assessment with Sonography for Trauma

US= ultrasound scan

\section{Declarations}

\section{Data availability}

The datasets generated during and/or analyzed during the current study are available from the corresponding author on reasonable request.

\section{Authors' contributions}

All the authors declare that they have all participated in the design, execution and analysis of the paper, and that they have approved the final version. Not any sentence is copied from other sources.

\section{Competing interests}

The authors declare no competing interests.

\section{References}

1. Mehta N, Babu S, Venugopal K. An experience with blunt abdominal trauma: evaluation, management and outcome. Clin Pract. 2014;4(2):599.

2. van der Vlies $\mathrm{CH}$, Olthof $\mathrm{DC}$, Gaakeer M, Ponsen $\mathrm{KJ}$, van Delden $\mathrm{OM}$, Goslings JC. Changing patterns in diagnostic strategies and the treatment of blunt injury to solid abdominal organs. Int $\mathrm{J}$ Emerg Med. 2011;4:47. 
3. Bahrami-Motlagh H, Hajijoo F, Mirghorbani M, SalevatiPour B, Haghighimorad M. Test characteristics of focused assessment with sonography for trauma (FAST), repeated FAST, and clinical exam in prediction of intra-abdominal injury in children with blunt trauma. Pediatr Surg Int. 2020;36(10):122734.

4. Gamanagatti S, Rangarajan K, Kumar A, Jineesh. Blunt abdominal trauma: imaging and intervention. Curr Probl Diagn Radiol. 2015;44(4):321-36.

5. Jakobsen JA, Oyen R, Thomsen HS, Morcos SK, Members of Contrast Media Safety Committee of European Society of Urogenital R. Safety of ultrasound contrast agents. Eur Radiol. 2005;15(5):9415.

6. Laugesen NG, Nolsoe CP, Rosenberg J. Clinical Applications of Contrast-Enhanced Ultrasound in the Pediatric Work-Up of Focal Liver Lesions and Blunt Abdominal Trauma: A Systematic Review. Ultrasound Int Open. 2017;3(1):E2-E7.

7. Holmes JF, Gladman A, Chang $\mathrm{CH}$. Performance of abdominal ultrasonography in pediatric blunt trauma patients: a meta-analysis. J Pediatr Surg. 2007;42(9):1588-94.

8. McGahan JP, Rose J, Coates TL, Wisner DH, Newberry P. Use of ultrasonography in the patient with acute abdominal trauma. J Ultrasound Med. 1997;16(10):653-62; quiz 63-4.

9. Pearce MS, Salotti JA, Little MP, McHugh K, Lee C, Kim KP, et al. Radiation exposure from CT scans in childhood and subsequent risk of leukaemia and brain tumours: a retrospective cohort study. Lancet. 2012;380(9840):499-505.

10. Scaife ER, Rollins MD, Barnhart DC, Downey EC, Black RE, Meyers RL, et al. The role of focused abdominal sonography for trauma (FAST) in pediatric trauma evaluation. J Pediatr Surg. 2013;48(6):1377-83.

11. Poletti PA, Platon A, Becker CD, Mentha G, Vermeulen B, Buhler LH, et al. Blunt abdominal trauma: does the use of a second-generation sonographic contrast agent help to detect solid organ injuries? AJR Am J Roentgenol. 2004;183(5):1293-301.

12. Sessa B, Trinci M, lanniello S, Menichini G, Galluzzo M, Miele V. Blunt abdominal trauma: role of contrast-enhanced ultrasound (CEUS) in the detection and staging of abdominal traumatic lesions compared to US and CE-MDCT. Radiol Med. 2015;120(2):180-9.

13. Valentino M, De Luca C, Galloni SS, Branchini M, Modolon C, Pavlica P, et al. Contrast-enhanced US evaluation in patients with blunt abdominal trauma(). J Ultrasound. 2010;13(1):22-7.

14. Callahan MJ, Poznauskis L, Zurakowski D, Taylor GA. Nonionic iodinated intravenous contrast material-related reactions: incidence in large urban children's hospital--retrospective analysis of data in 12,494 patients. Radiology. 2009;250(3):674-81.

\section{Figures}




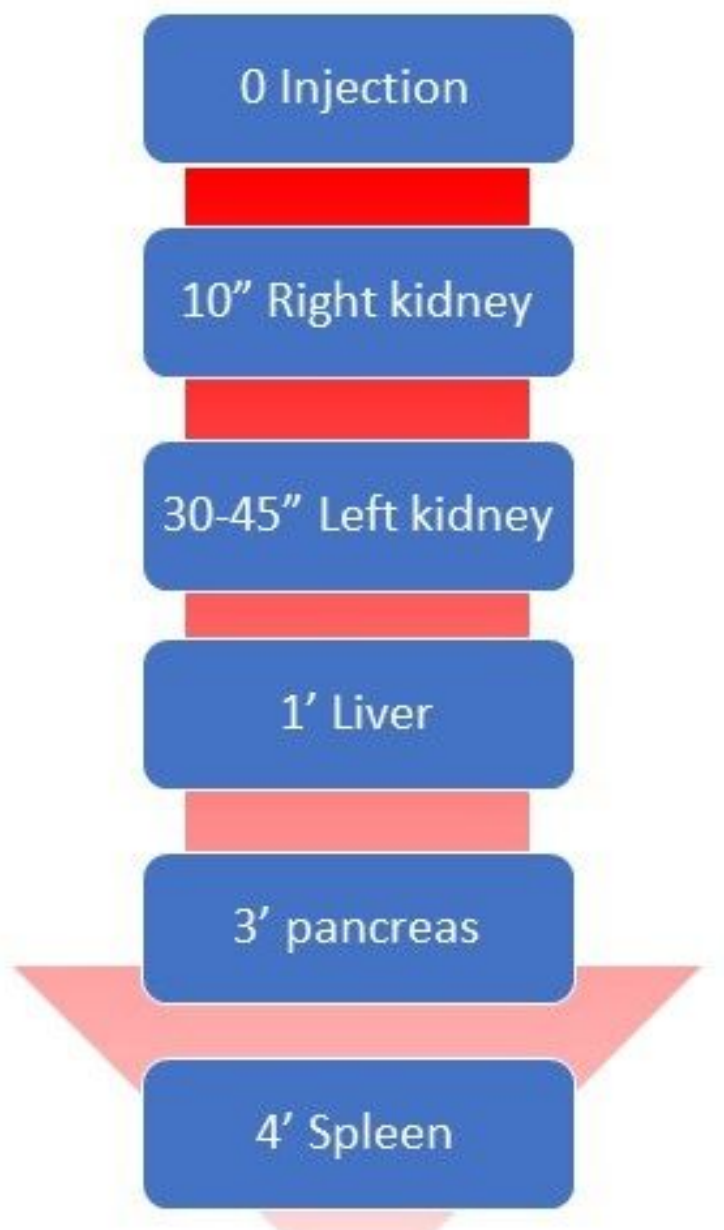

Figure 1

CEUS: time of organ's visualization after injection of intravenous contrast agent 


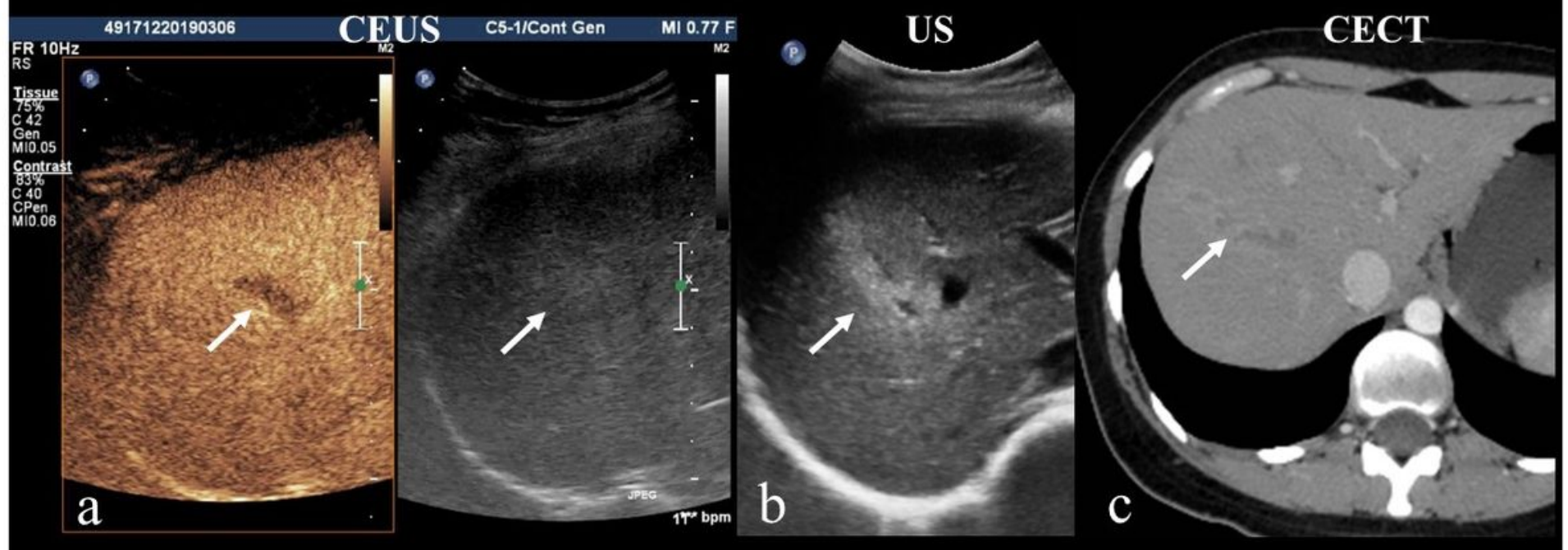

Figure 2

Patient No 4. US reveals normal spleen appearance with no peri-splenic fluid unlike the CEUS and CECT which depict splenic rapture (arrows) and an amount of peri-splenic fluid (stars).
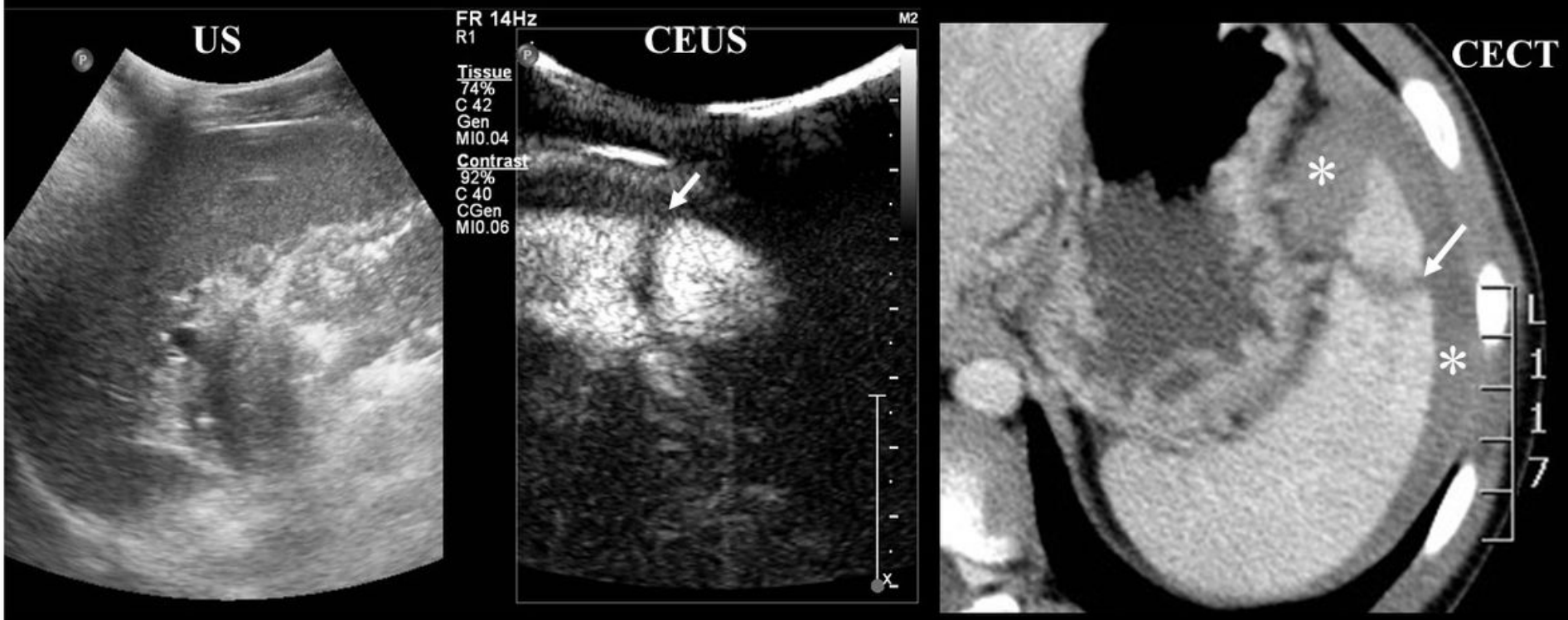


\section{Figure 3}

Patient No 11. A linear focal hyperattenuation on US confirmed as liver laceration both on CEUS and CECT (arrows). a: CEUS; side by side images. The B-Mode image (right image) is not sufficient for diagnostic purpose due to low mechanical index (MI) in order to prevent microbubbles, b: Initial Ultrasound (US), c: CECT 